\title{
Clinical Implication of Colorectal Cancer Stem Cells: Still Has a Long Way to Go
}

\author{
Hungdai Kim \\ Department of Surgery, Gastrointestinal Cancer Center, Kangbuk Samsung Hospital, Seoul, Korea
}

See Article on Page 84-91

Cancer stem cells (CSCs) have two distinct functional properties, the ability to perpetuate themselves throughout an extended period of time (self-renewal) and the potential to generate all the differentiated cells of the tissue of origin (multipotency). CSCs originate from normal stem cells after the accumulation of mutations, and growth-promoting signals change the microenvironment or niche for CSCs undergoing uncontrolled proliferation. In a clinical aspect, the main concern with CSCs is the underlying cause of treatment failure following surgery, chemotherapy with or without target agents, and radiotherapy. Drug failure occurs in $90 \%$ of metastatic cancers and is attributed to therapeutic resistance, which is associated with increased aerobic glycolysis, fatty acid synthesis, and glutamine metabolism, resulting in decreases in drug-induced apoptosis. Thus, the factors that sustain CSCs must be defined in order to develop more efficient therapeutics.

Although expression levels of CSC surface antigens are variable and their frequent coexpressions on normal stem cells have made CSC antigen distinction difficult, CD133, CD24, CD29, CD44, CD166 (ALCAM), EpCAM, Lgr5, ALDH1A1, and ALDH1B1 have been identified as potential markers for colon CSC. However, the best markers to identify CSCs have not yet been well defined. A CSC profile has been suggested to regulate key steps leading to progression, relapse and metastasis and may, therefore, be responsible for this unpredictable heterogeneity.

According to various studies, the usefulness of the expressions of CSC surface markers as predictors of patient prognosis is inconclusive because of contradictory data. Huang et al. [1] ob-

Correspondence to: Hungdai Kim, M.D.

Department of Surgery, Gastrointestinal Cancer Center, Kangbuk Samsung

Hospital, 29 Saemunan-ro, Jongno-gu, Seoul 110-746, Korea

Tel: +82-2-2001-8541, Fax: +82-2-2001-2131

E-mail: hungdai.kim@samsung.com

(c) 2015 The Korean Society of Coloproctology

This is an open-access article distributed under the terms of the Creative Commons Attribution NonCommercial License (http://creativecommons.org/licenses/by-nc/3.0) which permits unrestricted noncommercial use, distribution, and reproduction in any medium, provided the original work is properly cited. served that stem cells taken from colorectal cancer patients could be evaluated for gene expressions of CD44 and CD133 and that patients with tumors harboring higher levels of both CD44 and CD133 had a higher risk of developing early liver metastases. On the contrary, in a study by Dallas et al. [2], cells that were engineered to be knock-down for CD44 expression had almost a 10fold increase in metastatic potential in both the liver and the lungs. In addition to that, CD44 negative cells exhibited a greater "mechanical compliance", a property that is considered crucial in the process of extravasation and migration through the blood stream. These findings were similar with Hong et al. [3]'s recent findings that low levels of CD44 were relevant for increased tumor relapse and short disease-free survival in colon cancer patients. A possible explanation for this conflicting data could be that genetic and epigenetic differences have impacts on the expressions of CSC surface markers.

Giampieri et al. [4] assessed the role of colorectal CSC markers (ALCAM, CD133, CD24, LGR5, SOX2, and ALDH1A1) in determining the clinical outcomes for stage-III colon-cancer patients receiving adjuvant chemotherapy. They concluded that patients expressing high ALCAM levels may require more intensive treatment and possibly a change to a different treatment option.

Still many questions related to CSCs remain to be solved. Growth factors, such as epidermal growth factor (EGF) and vascular endothelial growth factor, and cytokines, such as transforming growth factor- $\beta$, tumor necrosis factor- $\alpha$, and interluekin- 6 , produced by a microenvironment can revert differentiated cells to a more stem-cell-like state and the EGF signaling pathway may regulates intestinal epithelial cell and stem/progenitor cell growth and differentiation. However, little knowledge exists concerning the role of growth factors in mediating proliferation and self-renewal of colon CSCs.

CSC numbers have been shown to be increased after chemotherapy or irradiation for the treatment of CRC [5]. Quiescent CSCs are spared by cytotoxic therapies, which usually result in a relative increase in tumor stem-cell content due to the selective survival of the CSC fraction. Moreover, after initially targeting proliferating CSCs, multiple cycles of chemotherapy may promote CSC proliferation and self-renewal. Besides chemotherapy, tar- 


\section{Coloproctologagy

geted therapies that partially or totally ablate the CSC pool may have to face the problem of CSC regeneration due to the ability of non-stem cells to recreate CSCs. In an even worse scenario, targeted therapies may elicit a reactive response resulting in the resurgence of more aggressive tumors. A possible solution for avoiding posttherapy regeneration of CSCs would be to combine CSC-targeted therapies with drugs that inhibit either the microenvironmental or the epigenetic mechanisms responsible for the reprogramming of transit-amplifying progenitors into CSCs. Interfering with tumor cell plasticity may, therefore, offer new tools to support the activity of both conventional and targeted anticancer drugs.

Further extensive studies are needed to elucidate whether these intestinal stem-cell markers can be used as predictive and prognostic biomarkers in a clinical setting. In addition, emerging CSCeliminating therapies should target molecular pathways in CSCs and mediators, as well as in their surrounding microenvironments.

\section{CONFLICT OF INTEREST}

No potential conflict of interest relevant to this article was reported.

\section{REFERENCES}

1. Huang X, Sheng Y, Guan M. Co-expression of stem cell genes CD133 and CD44 in colorectal cancers with early liver metastasis. Surg Oncol 2012;21:103-7.

2. Dallas MR, Liu G, Chen WC, Thomas SN, Wirtz D, Huso DL, et al. Divergent roles of CD44 and carcinoembryonic antigen in colon cancer metastasis. FASEB J 2012;26:2648-56.

3. Hong I, Hong SW, Chang YG, Lee WY, Lee B, Kang YK, et al. Expression of the cancer stem cell markers CD44 and CD133 in colorectal cancer: an immunohistochemical staining analysis. Ann Coloproctol 2015;31:84-91.

4. Giampieri R, Scartozzi M, Loretelli C, Del Prete M, Faloppi L, Bianconi $\mathrm{M}$, et al. Cancer stem cells profile and clinical outcome in stage III colon cancer patients receiving adjuvant oxaliplatinbased chemotherapy [abstract]. In: Proceedings of the 2014 Gastrointestinal Cancers Symposium; 2014 Jan 16-18; San Francisco, CA, USA. Alexandria (VA): American Society of Clinical Oncology; 2014. Abstract No. 474.

5. Dylla SJ, Beviglia L, Park IK, Chartier C, Raval J, Ngan L, et al. Colorectal cancer stem cells are enriched in xenogeneic tumors following chemotherapy. PLoS One 2008;3:e2428. 